$\begin{array}{cl}\begin{array}{c}\text { Revue } \\ \text { de } / \text { histoire } \\ \text { des religions }\end{array} & \text { Revue de l'histoire des religions } \\ & \begin{array}{l}\mathbf{4} 2012 \\ \text { Varia }\end{array}\end{array}$

\title{
Le Jubilé Loisy de 1927. Entre histoire des religions et histoire du christianisme
}

The Loisy Congress of 1927: Between history of religion and history of Christianity

\section{Annelies Lannoy}

\section{OpenEdition}

\section{Journals}

Édition électronique

URL : http://journals.openedition.org/rhr/7981

DOI : $10.4000 /$ rhr.798

ISSN : 2105-2573

Éditeur

Armand Colin

Édition imprimée

Date de publication : 1 décembre 2012

Pagination : 503-526

ISBN : 978-2200-92796-7

ISSN : 0035-1423

\section{Référence électronique}

Annelies Lannoy, "Le Jubilé Loisy de 1927. Entre histoire des religions et histoire du christianisme »,

Revue de l'histoire des religions [En ligne], 4 | 2012, mis en ligne le 01 décembre 2015, consulté le 03 mai 2019. URL : http://journals.openedition.org/rhr/7981 ; DOI : 10.4000/rhr.7981 


\section{Le Jubilé Loisy de 1927 Entre histoire des religions et histoire du christianisme}

Du 19 au 22 avril 1927 se tint à Paris le Congrès d'histoire du christianisme en l'honneur d'Alfred Loisy, qui fêtait alors ses 70 ans. Franz Cumont, ami de Loisy depuis longtemps, qui appartenait au Comité d'honneur du Congrès, retira finalement son adhésion. À la recherche des raisons du désengagement de Cumont, nous avons étudié la correspondance entre Loisy, Cumont et Paul-Louis Couchoud, organisateur du Congrès. Nous nous trouvons ainsi confrontés à une discussion sur l'aptitude professionnelle et l'identité idéologique du chercheur actif dans le domaine de l'histoire du christianisme en France durant la première moitié $d u X^{e}$ siècle, un débat lié à la question de la position de l'étude du christianisme au sein de l'histoire des religions.

\section{The Loisy Congress of 1927: Between history of religion and history of Christianity}

From April 19th to 22nd 1927, the Congrès d'histoire du christianisme was held in honor of Alfred Loisy, who was celebrating his 70th birthday at the time. At first Franz Cumont, Loisy's longtime friend, belonged to the congress's Honorary Committee, but ultimately he withdrew his support. In search of the reasons for this withdrawal, I study the correspondence between Loisy, Cumont and Paul-Louis Couchoud, organizer of the event. I thus discover a discussion about the competence and ideological stance of the researcher active within the field of the history of Christianity in France in the first half of the $20^{\text {th }} C$, a debate closely related to the question of the position of the study of Christianity within the discipline of the history of religion. 
Comme l'ont montré Émile Poulat, François Laplanche et bien d'autres savants, en France l'institutionnalisation de l'histoire des religions s'est accomplie dans une atmosphère tendue du point de vue confessionnel. Au cours des années 1880, les premières chaires séculières d'histoire des religions sont créées à l'issue de la suppression des facultés d'État de théologie'. Les titulaires des nouvelles chaires se proposent d'étudier l'histoire du christianisme d'un point de vue non-confessionnel et scientifique. Dans les milieux français catholiques, ces changements ne reçoivent pas un accueil favorable. Ils contribuent à une séparation progressive entre l'histoire des religions laïque et l'histoire religieuse pratiquée dans les Instituts catholiques, créés sur-le-champ à la suite de la suppression des facultés de théologie. En outre, la redéfinition de la position de l'histoire du christianisme au sein de la discipline «histoire des religions» provoque des discussions intenses dans le camp laïc sur les méthodes à suivre et la définition d'une approche neutre et scientifique. Essentiellement, tous ces débats remontent à une question élémentaire : le christianisme est-il une religion parmi d'autres?

En 1909, le nouveau titulaire de la chaire d'histoire des religions au Collège de France fournit, dans sa leçon inaugurale, la preuve des désaccords existants ${ }^{2}$. Alfred Loisy, ancien prêtre, excommunié pour modernisme à peine une année auparavant, $\mathrm{y}$ prend ses distances par rapport aux approches, à son avis insuffisamment impartiales, de ses prédécesseurs protestants, Albert et Jean Réville.

Franz Cumont écrivit à Loisy pour le féliciter de sa nomination au Collège de France et cette lettre fut le point de départ d'une

1. À l'occasion des centenaires de ces chaires, les études consacrées à l'institutionnalisation se sont multipliées. Voir la bibliographie de François Laplanche, La crise de l'origine, Paris, Albin Michel, 2006, p. 25 note 2 - désormais abrégé F. Laplanche (2006). Pour ce qui suit: Émile Poulat, «L'institution des sciences religieuses», in Jean Baubérot (éd.), Cent ans de sciences religieuses en France, Paris, Cerf, 1987, p. 49-78; et François Laplanche, «L'histoire des religions en France au début du $\mathrm{xx}^{\mathrm{e}}$ siècle», Mélanges de l'École Française de Rome t.111/2 (1999), p. 623-634.

2. Alfred Loisy, Leçon d'ouverture du cours d'histoire des religions au Collège de France, Paris, Émile Nourry, 1909. 
correspondance abondante ${ }^{3}$. Se suivant de manière presque ininterrompue jusqu'à la mort de Loisy en 1940, ces lettres nous offrent un regard unique sur l'évolution de la jeune discipline. Au cours des années 1926-1927, on peut y suivre en particulier les préparatifs du Congrès d'histoire du christianisme, qui se tint du 19 au 22 avril 1927 au Collège de France en l'honneur d'Alfred Loisy; celui-ci fêtait alors ses soixante-dix ans. Par ces lettres, ainsi que par les Mémoires de Loisy et l'annonce du Congrès dans la Revue de l'histoire des religions, on apprend qu'un Comité d'honneur fut créé pour l'occasion, réunissant toute une série de personnalités internationales dans le domaine des sciences religieuses ${ }^{4}$. Les actes du Congrès, publiés en 1928, mentionnent par exemple Adolf von Harnack et Carl Clemen qui y représentaient l'Allemagne; pour l'Angleterre, il y avait entre autres Sir James Frazer; pour l'Italie, Ernesto Buonaiuti et Raffaele Pettazzoni ; parmi les compatriotes de Loisy, on signalera Charles Guignebert et Salomon Reinach ${ }^{5}$. La correspondance CumontLoisy et les Mémoires nous apprennent qu'à l'origine Franz Cumont avait également fait partie du Comité d'honneur'. Mais en fin de compte, son nom n'apparaît nulle part dans la publication des actes du Congrès : ni dans le Comité d'honneur, ni dans la liste des conférenciers.

D'après les Mémoires de Loisy, le désengagement de Cumont s'expliquerait par sa défiance à l'égard des organisateurs du Congrès : «Mon savant ami Franz Cumont avait été mis en inquiétude au sujet de ce congrès par certaines personnes, (...) et, le 3 juillet 1926,

3. La correspondance Cumont-Loisy est conservée à la Bibliothèque nationale de France, à l'exception de 9 lettres de Loisy conservées à l'Academia Belgica à Rome. Le dossier compte environ 171 lettres de la main de Loisy et 233 de la main de Cumont. Lettres de Cumont à Loisy: BnF, ms. NAF 15651, ff. 64-442 (mai 1909-10 mai 1940); lettres de Loisy à Cumont: BnF, ms. NAF 15644, ff. 55-329 (8 mai 1908-8 janvier 1940). La publication est prévue dans un avenir proche, par les soins d'Aline Rousselle, Corinne Bonnet, Danny Praet, Sarah Rey et Annelies Lannoy.

4. Alfred Loisy, Mémoires pour servir à l'histoire religieuse de notre temps, Paris, Émile Nourry, 1931, vol. III, p. 490-491 - abrégé désormais A. Loisy (1931); et «Jubilé Alfred Loisy et Congrès d'Histoire du Christianisme», Revue de l'histoire des religions (désormais abrégée RHR) t. 93 (1926), p. 176.

5. Paul-Louis Couchoud (éd.), Jubilé Alfred Loisy. Congrès d'histoire du Christianisme, Paris-Amsterdam, Rieder, 1928 (3 v.); Marguerite Brunot, «Le congrès d'histoire du christianisme», RHR t. 96 (1927), p. 39-49.

6. A. Loisy (1931), p. 491. 
il m'écrivait pour me détourner d'une entreprise suspecte, lancée, disait-on, par un dilettante, pour son propre avantage et à mon réel détriment ${ }^{7}$.» Un bienheureux hasard a fait que les lettres que l'initiateur du congrès, Paul-Louis Couchoud, a écrites à Loisy ont été conservées, de même que ses lettres à Cumont. En outre, nous disposons du brouillon d'une lettre de Cumont à l'organisateur ${ }^{8}$. Les lettres de Loisy et de Cumont à Couchoud n'ont probablement pas été conservées. Au moyen de ces documents inédits, on découvrira que l'absence de Cumont n'était pas seulement motivée par les rumeurs sur le dilettantisme de l'organisateur, mais qu'un désaccord de fond sur l'identité idéologique des chercheurs qui s'occupaient alors de l'histoire du christianisme était tout autant à la base de l'attitude de Cumont.

Dans le présent article, nous nous proposons d'analyser un épisode des discussions sur l'histoire laïque du christianisme en France dans la première moitié $\mathrm{du} \mathrm{Xx}^{\mathrm{e}}$ siècle, à savoir le débat sur le rapport entre l'aptitude professionnelle et l'attitude idéologique du chercheur actif dans ce domaine. Étant donnée la professionnalisation progressive de l'histoire des religions à l'époque, des chercheurs de toute formation intellectuelle disposent-ils des compétences nécessaires pour l'étude de l'histoire du christianisme? De plus, les glissements institutionnels et méthodologiques suscitaient des questions sur la nécessité d'une certaine empathie envers le sujet d'étude, c'est-à-dire envers le christianisme, ce qui pouvait impliquer des restrictions à l'égard des chercheurs'. C'est en reconstruisant les rapports triangulaires entre les protagonistes du Congrès d'histoire du christianisme qu'il nous sera possible de découvrir l'éclatement des positions prises par les savants laïcs actifs en France dans l'histoire du christianisme et de préciser la position de cette dernière au sein de l'histoire des religions.

7. A. Loisy (1931), p. 496.

8. Les lettres de Couchoud à Loisy sont conservées à la Bibliothèque nationale: BnF, ms. NAF 15651, ff. 14-50 (11 avril 1920-4 juin 1938). Les lettres de Couchoud à Cumont se trouvent à l'Academia Belgica. L'ensemble des lettres reçues par Cumont est à consulter sur Internet au moyen de la base de données créée par C. Bonnet (http://www.academiabelgica.it/acadbel/askFCnew.php).

9. A. Loisy, Leçon d'ouverture..., p. 21 ; E. Poulat, op. cit. p. 67 et 77. 


\section{LE CONTEXTE DES ANNÉES 1920}

$\mathrm{Au}$ sortir de la Première Guerre mondiale, les réseaux personnels et les collaborations scientifiques préexistants étaient profondément perturbés. La série des Congrès d'histoire des religions, qui avaient eu lieu régulièrement depuis 1897, fut brusquement interrompue ${ }^{10}$. En France, la première initiative pour la création d'une nouvelle plateforme visant à renouer les relations intellectuelles brisées date de 1923. Cette année-là, la Société Ernest Renan organisa un congrès pour célébrer le centenaire d'Ernest Renan, sur la mémoire duquel elle veillait. La Société avait été fondée en 1919 par Paul Alphandéry et René Dussaud, les co-directeurs de la Revue de l'histoire des religions à l'époque, dans laquelle les comptes rendus des séances de la Société furent publiés jusqu'en $1983^{11}$. Elle se fixa surtout des objectifs de diffusion de la science religieuse française laïque. Orientée vers les libres-penseurs de l'intelligentsia française, la Société réunissait les plus illustres savants de France et d'ailleurs, parmi lesquels Alfred Loisy et Franz Cumont. Le congrès, tenu à la Sorbonne en octobre 1923, fut un succès et attira un public international. Pourtant, deux groupes de savants furent absents. À cause des séquelles respectives de la guerre, et de la crise moderniste dans l'Église catholique, ni les scientifiques allemands, ni les savants catholiques ne participèrent au congrès.

Le Congrès d'histoire du christianisme associé au Jubilé12 de Loisy se situe chronologiquement entre le congrès de 1923 et la reprise officielle des Congrès d'histoire des religions en 1929 à

10. Paul Alphandéry, «À propos du récent Congrès d'Histoire des religions », RHR t. 100 (1929), p. 205-218 et Arie L. Molendijk, «Les premiers congrès d'histoire des religions, ou comment faire de la religion un objet de science?», Revue germanique internationale $\mathrm{n}^{\circ} 12$ (2010), p. 91-103. Dans la correspondance de Cumont, plusieurs contacts ne seront pas entretenus pendant la guerre: Corinne Bonnet, Le "grand atelierde la science . Franz Cumont et l'Altertumswissenschaft. Héritages et émancipations. Des études universitaires à la fin de la Première Guerre mondiale (1888-1923), Bruxelles - Rome, Brepols, 2005, p. 300 sq. (v. I).

11. Sur la Société Renan et la publication du Bulletin de la Société Ernest Renan dans la $R H R$, voir F. Laplanche (2006), p. 92-95. L'élection de Cumont est annoncée au cours de la séance du 27 décembre 1920: RHR t. 83 (1921), p. 236.

12. Nous avons opté pour la graphie avec majuscule initiale, conforme à la plupart des sources citées, sauf dans le cas de Cumont pour lequel nous avons maintenu la minuscule. 
Lund. L'initiative venait de Paul-Louis Couchoud, qui est entré dans l'histoire comme un des défenseurs français de la théorie dite «mythique ${ }^{13}$ ». Les adeptes de cette théorie, appelés «les mythologues» par Loisy et Cumont, nient l'existence historique de Jésus-Christ. Ils pensent, à l'opposé, que les évangiles sont les récits de la vie d'un dieu, d'un personnage entièrement mythique. Ce furent surtout les publications de Couchoud qui firent éclater la discussion sur la thèse «mythique» en France dans toute sa violence. Les remous provoqués par son article «L'Énigme de Jésus» en 1923 et son livre Le mystère de Jésus en 1924, dans lesquels il conclut que le personnage historique de Jésus n'a jamais existé, furent considérables ${ }^{14}$. Pour l'histoire du Congrès d'histoire du christianisme, il importe de souligner que les débats français sur la nature de Jésus, engagés en grande partie dans la Revue de l'histoire des religions, devinrent la proie d'une bipolarisation confessionnelle, la thèse mythique se trouvant des alliés de poids dans le camp laïc, dont Prosper Alfaric. De plus, en 1923 Couchoud avait fondé la collection «Christianisme» qui se proposait de diffuser auprès du grand public les travaux récents sur le christianisme et son histoire. Couchoud convainquit Joseph Turmel, qui publiait sous le pseudonyme de «Henri Delafosse», de collaborer à sa série; il gagna à son entreprise des savants comme l'ex-prêtre Albert Houtin, Ernesto Buonaiuti et même l'adversaire le plus sévère de sa thèse mythique, Loisy ${ }^{15}$. Par une lettre datée du 28 novembre 1923, on apprend que Couchoud invita aussi Cumont à collabore ${ }^{16}$ : il lui proposait de publier une édition abrégée de Les religions orientales dans le paganisme romain, ce qui démontre une fois de plus que le livre de Cumont était perçu comme d'une importance capitale pour l'histoire du christianisme, même si la religion chrétienne n'y était mentionnée qu'obliquement. La lettre de Cumont n'étant pas conservée, on ne saura jamais quelle a été sa réponse, mais le fait est que cette édition ne vit pas le jour.

13. Maurice Goguel, «Recent French Discussions of the Historical Existence of Jesus Christ», Harvard Theological Review t. 20 (1927), p. 115-142; Alan H. Jones, Independence and exegesis, Tübingen, J.C.B. Mohr (Paul Siebeck), 1983, p. 105-113 - abrégé désormais A.H. Jones (1983).

14. Paul-Louis Couchoud, Le Mystère de Jésus, Paris, 1924, et «L’Énigme de Jésus », Mercure de France ${ }^{\circ}$ CLXII (1923), p. 344-466.

15. Voir A. H. Jones (1983), p. 108; M. Goguel, op. cit. p. 64-65.

16. Couchoud à Cumont, 28/11/1923, cote 739 (Academia Belgica). 
La théorie mythique de Couchoud et de ses partisans se heurtait à de grandes résistances. Dans les milieux non catholiques, ce fut d'abord Charles Guignebert qui publia un compte rendu destructeur du Mystère de Jésus ${ }^{17}$, puis le protestant libéral Maurice Goguel ${ }^{18}$ et Loisy qui engagèrent le débat. Dénonçant sa qualité de «philosophe et médecin épris d'exégèse ${ }^{19}$ », ils reprochèrent à Couchoud, médecin personnel d'Anatole France, son incompétence exégétique et historique. Dans l'œuvre de Loisy, la réfutation de la thèse constitue d'ailleurs un véritable fil conducteur $^{20}$. La première publication dans laquelle Loisy s'oppose à la non-historicité de Jésus remonte à 1910; son avant-dernier livre: Autres mythes à propos de la Religion (1938), reprend ses arguments principaux.

Dans l'œuvre publiée de Cumont, on ne trouve aucune référence à la discussion mythique. Cela s'explique par le contenu spécifique des publications de Cumont en tant qu'expert des religions païennes. $\mathrm{Vu}$ la connotation confessionnelle du débat relatif à l'influence exercée par les mystères et les «religions orientales» sur le christianisme, il décida de bonne heure de ne pas se mêler des querelles sur l'origine du christianisme ${ }^{21}$. Cela n'implique pas pour autant qu'il n'ait pas eu d'opinion sur la question de l'historicité de Jésus ou qu'il ait nié la réalité des liens entre le christianisme et les religions païennes. Dans ses lettres adressées à Loisy, Cumont ne mâche pas ses mots en exprimant ce qu'il pense de la thèse mythique. Ainsi, il écrit le 27 janvier 1922: «Il faut avoir le cerveau embrumé par l'abus de la théologie ou faussé par des préjugés doctrinaires pour refuser de reconnaître à l'origine du christianisme cette action d'une personne réelle sans laquelle il reste une énigme ${ }^{22}$.» Les mots fermes de Cumont critiquent les positions ultras du camp chrétien et

17. Charles Guignebert, «Couchoud (P. L.). Le Mystère de Jésus », RHR t. 94 (1926), p. 215-244.

18. Maurice Goguel réagit avec son article «À propos de "L'énigme de Jésus" », Mercure de France ${ }^{\circ}$ CLXIV (1923), p. 389-404.

19. C. Guignebert, op. cit. p. 215.

20. Voir A. H. Jones (1983), p. 105-113 pour la position de Loisy envers «les mythologues».

21. P. ex. Franz Cumont, Les religions orientales dans le paganisme romain, éd. Corinne Bonnet - Françoise Van Haeperen, Torino, Brepols-Aragno, 2006, p. 6. Voir aussi Corinne Bonnet, «Franz Cumont et les risques du métier d'histoire des religions », Hieros t. 5 (2000), p. 12-29.

22. Cumont à Loisy, 27/01/1922, BnF ms. NAF 15651, fo 318. 
du camp non-confessionnel, l'un comme l'autre menant, à son avis, à la falsification du débat.

\section{Scepticisme et doutes chez Cumont et Loisy}

Lorsque Couchoud, tant de fois attaqué par Loisy pour ses positions scientifiques, écrit le 10 février 1926 à Cumont pour le renseigner sur le projet du «Jubilé Loisy» et lui demander son adhésion au Comité d'honneur du Congrès d'histoire du christianisme, Cumont se tient sur ses gardes. Couchoud lui présente le Congrès comme l'initiative concertée des élèves et des collègues de Loisy au Collège de France, par lesquels il est chargé, en tant que secrétaire du Comité d'organisation, de prendre contact avec les savants pressentis pour le Comité d'honneur. La correspondance de Cumont et Loisy montre que Cumont préféra consulter Loisy avant de prendre une décision; il lui écrivit le 13 février:

Je reçois de Couchoud la demande de faire partie du comité d'un Congrès d'histoire du christianisme qui serait organisé l'an prochain en votre honneur. Bien que je ne me sois jamais occupé de l'histoire susdite qu'en amateur, je donnerai volontiers mon adhésion, s'il agit [sic] de témoigner de mon estime et de ma sympathie. Envoyez-moi donc un mot pour me donner l'assurance que ce congrès a votre haute approbation. Il ne faudra pas moins que cette certitude pour m'engager à accepter un patronage pour lequel je me sens peu qualifié par mes études païennes ${ }^{23}$.

Même si, en réalité, beaucoup de ses «études païennes » portaient sur l'histoire du christianisme ou avaient des répercussions sur celle$\mathrm{ci}^{24}$, Cumont distingue ici nettement les deux domaines. À ce point de la correspondance, il est difficile de déterminer si les termes de Cumont sont réellement inspirés par un scrupule scientifique. En soulignant la divergence d'avec ses propres «études païennes», il se pourrait aussi qu'il essayât de prendre déjà ses distances par rapport au Congrès et, comme nous le supposons, par rapport à son organisateur.

Dans sa réponse du 16 février, Loisy procure à Cumont des renseignements sur l'entreprise. L'initiative ne vient pas de

23. Cumont à Loisy: BnF, ms. NAF 15651, fo 357.

24. Par exemple son article «Les actes de S. Dasius», Analecta Bollandiana t. XVI (1897), p. 5-16, qui a provoqué une discussion sur Jésus comme «Roi des Saturnales». 
Couchoud, mais du personnage qui avait financé le congrès de la Société Renan en 1923, et dont Loisy ne révèle pas l'identité. Les Mémoires et les lettres de Couchoud à Loisy nous apprennent qu'il le connaissait pourtant fort bien: il s'agit de Jean Leclerc de Pulligny, ingénieur de profession et actif dans la haute administration. En tant que franc-maçon de tendance rationaliste, Pulligny s'intéressait vivement à l'histoire des religions ${ }^{25}$. Dans sa lettre, Loisy explique aussi pourquoi on avait opté pour un congrès d'histoire du christianisme et pour l'association avec son Jubilé:

Ne voyant pas possibilité de réunir un nouveau congrès d'histoire des religions, on a songé à un congrès d'histoire du christianisme; je crois savoir que l'idée a été d'abord assez froidement accueillie dans le milieu parisien; puis les initiateurs ont eu l'idée d'y accrocher les soixante-dix ans; c'est alors que j'ai eu connaissance de leur projet ${ }^{26}$.

En 1926, l'ancien comité qui était en charge, avant la guerre, de l'organisation des Congrès d'histoire des religions n'était toujours pas opérationnel ${ }^{27}$. C'est pourquoi on avait formé le projet du Congrès de l'histoire du christianisme. Loisy suggère toutefois que ce n'était pas si simple. Aux Congrès d'histoire des religions, l'histoire du christianisme était une section parmi d'autres, ce qui doit être compris comme une prise de position sur la possibilité de comparer le christianisme avec d'autres religions. L'idée de rendre autonome la section de l'histoire du christianisme pourrait logiquement donner l'impression d'une prise de position inverse, impliquant que le christianisme doit recevoir un traitement à part. C'est probablement pourquoi le projet ne trouva pas un accueil favorable «dans le milieu parisien». Ensuite, les organisateurs avaient songé y rattacher le Jubilé de Loisy, lequel étudiait l'histoire du christianisme de manière comparée et non dogmatique. Ainsi les organisateurs trouveraient le moyen non seulement d'exciter l'intérêt du grand public, mais aussi de diminuer les risques d'un malentendu idéologico-confessionnel. Pour le reste, Loisy se montra réservé et même sceptique envers l'idée du Congrès:

Je n'ai pas cru pouvoir leur opposer un refus; mais j'ai dit à Couchoud: "Vous allez recruter au moins quatre adhérents.» J'attends avec une certaine curiosité les réponses qui seront faites.

25. A. H. Jones (1983), p. 44.

26. Loisy à Cumont, 16/02/1926, cote 7991 : Academia Belgica.

27. A. Loisy (1931), p. 535. 
Car on a pensé à un comité international. Je ne crois pas que vous risquiez rien en donnant votre adhésion. Je ferai mon possible pour que ce congrès n'ait aucunement l'air d'une manifestation anticléricale ou anticatholique. D'ailleurs, il se tiendrait au Collège de France, et l'administrateur y a donné son approbation ${ }^{28}$.

Sa promesse de veiller à l'atmosphère impartiale du Congrès indique d'ailleurs que Loisy était conscient des associations particulières qu'évoquait le nom de Couchoud, du reste probablement suggérées par Cumont dans sa lettre prudente.

Ajoutons, pour la compréhension de la psychologie de Loisy, que sur cette affaire la correspondance n'est pas tout à fait en accord avec les Mémoires. Comparé à la lucidité avec laquelle il comprend les restrictions implicites de la lettre de Cumont, Loisy y apparaît comme remarquablement naïf :

Je ne m'arrêtai pas à considérer que la personnalité de Couchoud, connu surtout par l'éclat de son Mystère, pouvait donner à l'affaire une couleur inquiétante, ni que le congrès pourrait avoir été conçu en réclame pour le Mystère. Ne croyant pas à la réussite et ne voulant pas désobliger Couchoud, qui, en 1921, était venu en aide à la Revue d'histoire et littérature religieuses en se chargeant de cent abonnements, je lui permis de sonder le terrain ${ }^{29}$.

Couchoud lui ayant rendu service pour la situation pénible de la $R H L R$ après la guerre, Loisy aurait été prêt à l'aider en retour sans aucunement prendre en considération les racines anticléricales de Couchoud ni son opinion particulière sur le sujet même du Congrès, l'histoire du christianisme. La dissonance entre la correspondance et les Mémoires fait donc émerger la position ambiguë de Loisy qui, d'une part, ne croit pas à la réussite du Congrès mais fait quand même son possible pour le promouvoir, et qui, d'autre part, ne doute pas des bonnes intentions de Couchoud mais promet à Cumont de ne pas laisser dégénérer le projet en une manifestation anticatholique ou anticléricale.

28. Loisy à Cumont, 16/02/1926, cote 7991 : Academia Belgica. Les italiques correspondent aux passages soulignés par Loisy. L'Administrateur, Maurice Croiset, était membre de la Société Ernest Renan: Laplanche (2006), p. 93.

29. A. Loisy (1931), p. 490. 


\section{UN ENJEU CACHÉ : CONTRE LA «THÈSE MYTIQUE» DE COUCHOUd}

À en croire Loisy, les premiers nuages s'accumulent au-dessus du Congrès vers la fin du mois de mars ${ }^{30}$. Le 31 mars 1926, il reçoit un visiteur à Ceffonds qui lui fait part de son inquiétude au sujet du projet:

Un visiteur des mieux intentionnés m'était venu dire que des bruits singuliers, dont tels notables personnages se faisaient l'écho, couraient à ce sujet: on prétendait que le Dr Couchoud ne faisait le congrès que pour lui, pour la réclame de son Mystère, et que je donnerais ma démission, après le congrès, en désignant Couchoud pour mon successeur au Collège de France ${ }^{31}$.

Loisy avait effectivement décidé de se faire suppléer au Collège de France à la rentrée suivante, tout en conservant sa chaire. Cette suppléance signifiait une période de repos par rapport à sa double charge d'enseignement. En 1924, à l'âge de soixante-six ans, il avait également accepté celle de Religion d'Israël à l'École pratique des Hautes Études. Selon Loisy, la rumeur concernant Couchoud était absurde puisqu'il avait désigné dès le mois d'avril 1925 le spécialiste du mysticisme chrétien, Jean Baruzi, pour le remplacer. $\mathrm{Du}$ reste, le passage pertinent des Mémoires reste bien obscur. D'après Loisy, son visiteur faisait inconsciemment le jeu des initiateurs des rumeurs qui, en les répandant, voulaient surtout faire obstacle au Congrès.

Malgré sa défiance et son scepticisme initiaux à l'égard de la réussite du Congrès, Loisy ouvrit une enquête personnelle au cours des mois suivants pour démasquer les agents de la diffusion des rumeurs, mais également pour vérifier la fiabilité de Couchoud. Le premier qu'il mit dans la confidence sur ses hypothèses fut Albert Houtin. Houtin répondit négativement à la lettre de Loisy: ne connaissant guère Couchoud, il n'était pas au courant des rumeurs autour du Congrès ${ }^{32}$. Loisy décida alors de faire appel à un ami qu'il savait très lié au milieu parisien comme membre de l'Académie des Inscriptions et Belles Lettres (depuis 1904) et de la Société Ernest Renan (depuis 1920). Le 30 juin, il adressa à Franz Cumont ses

30. D'après les témoignages donnés dans les Mémoires: A. Loisy (1931), p. 494.

31. A. Loisy (1931), p. 494.

32. A. Loisy (1931), p. 494. 
nouvelles questions concernant la perception du Congrès. Cette fois, il se concentra sur le projet de la Société Renan d'organiser une suite au Congrès organisé en $1923^{33}$ :

J'ignore si le projet (Couchoud), de congrès d'histoire du christianisme est en concurrence ou non avec les intentions de la Société. Si la Société veut faire un Congrès d'histoire des religions en 1927, le Congrès-Christianisme n'a aucune raison d'être; et même si le Congrès général est pour 1928, le Congrès-Christianisme 1927 est plutôt contre-indiqué. Je n'ai rien su des intentions de la Société; j'ai su seulement, et pas par Couchoud, que la Société, comme telle, n'avait pas agréé le projet de Congrès-Christianisme. Ne pourriezvous pas m'obtenir des renseignements sûrs touchant les points que je viens d'indiquer ${ }^{34}$ ?

Apparemment, dès le milieu des années 1920 plusieurs organismes jugeaient le moment venu de renouer les liens au sein de la communauté scientifique internationale. Loisy lui-même était membre du Comité de la Société Renan, mais, selon ses dires, sa mauvaise santé l'avait empêché d'assister aux réunions ${ }^{35}$. Pourtant, la crainte de la rivalité que pourrait générer la poursuite du Congrès de l'histoire du christianisme ne constitua pas le seul motif de Loisy pour demander conseil à Cumont. Peut-être commençait-il aussi à perdre la confiance qu'il avait initialement en Couchoud. Le soulignage dans le texte («pas par Couchoud») trahirait alors son agacement face au silence de ce dernier sur les projets de la Société.

Franz Cumont répondit aussitôt. Le 3 juillet, il confirmait à Loisy qu'il y avait effectivement un Congrès d'histoire des religions en voie de préparation, à l'initiative de Nathan Söderblom, archevêque luthérien d'Uppsala. La Société Renan n'était pas encore officiellement au courant, mais elle serait favorable au projet. À ces renseignements factuels, Cumont joignait ses réflexions personnelles sur le Congrès :

Un «mythologue» est l'homme le moins indiqué pour faire réussir un pareil projet. La Société Renan, sollicitée, s'abstiendra et l'on m'assure qu'il en sera de même de la Faculté de théologie protestante

33. La section d'histoire du christianisme avait également occupé une place éminente au congrès de 1923: P. Alphandéry, op. cit. p. 210-211.

34. Loisy à Cumont, 30/06/1926, BnF, ms. NAF 15644, f $\mathrm{f}^{\circ} 280$.

35. A. Loisy (1931), p. 446. Les comptes rendus publiés par la RHR montrent que Cumont en revanche assistait fréquemment aux séances, lors de ses passages à Paris, et qu'il était présent à celle du 26 juin 1926 (RHR t. 95, 1927, p. 336), comme d'ailleurs Couchoud. 
de Strasbourg. Voici donc une réunion où l'on devra s'occuper de l'histoire du christianisme mais dont seront exclus la grande majorité de ceux qui s'intéressent à cette histoire, je veux dire les catholiques et les protestants orthodoxes. En revanche on y verra quelques théologiens laïcs qui viendront y prêcher leur foi négative. Beaucoup de libres esprits, comme les chefs de la Société Renan, hésitent à se compromettre en compagnie de gens qui chercheront certainement surtout à faire une manifestation anticléricale. Pour vous dire toute ma pensée, je crois que les promoteurs du Congrès se servent de votre nom illustre pour des fins que vous n'approuveriez certainement pas $^{36}$.

Dans cette lettre, les doutes plutôt prudents de Loisy se trouvent transformés en réalité choquante. Cumont y déclare franchement que la Société Ernest Renan, la Faculté de théologie protestante de Strasbourg et aussi lui-même ne peuvent faire abstraction des convictions supposées anticléricales des organisateurs. Rejetant toute forme de recherche partiale basée sur des préjugés dogmatiques, les «libres esprits» membres de la Société se distancieraient de l'anticléricalisme militant qu'ils pourraient détecter chez les organisateurs du Congrès. Le bulletin de la Société démontre pourtant que Couchoud et Pulligny assistèrent souvent aux séances. La thèse mythique de Couchoud y reçut beaucoup d'attention. Elle fut amplement discutée et aussi réfutée par deux autres membres bien connus de la Société, Maurice Goguel et Charles Guignebert. Il importe donc de ne pas considérer la Société comme un espace homogène. Les «chefs» auxquels Cumont se réfère dans sa lettre pourraient avoir été Alphandéry, fondateur de la Société qui sera nommé plus loin dans la correspondance, et Guignebert, président en 1922 et en 1928. Néanmoins, il reste difficile de déterminer avec certitude quels étaient les autres hommes de poids dans la Société.

Pour Cumont, le terme de «mythologue», dans ce contexte, n'équivaut plus seulement à une simple conviction sur la nonhistoricité de Jésus Christ, il indique aussi la partialité anticléricale de «l'homme» en question. Cumont souligne les conséquences pour les collègues catholiques et protestants orthodoxes qui s'excluront inévitablement d'une telle entreprise. En réalité, cette attitude plutôt favorable à ces derniers pourrait apparaître comme une rupture dans les convictions de Cumont. Lui-même avait eu à souffrir du parti catholique en Belgique, lorsque le ministre (catholique) des Arts 
et des Sciences, dont dépendait la nomination des professeurs dans les universités d'État, avait refusé de lui attribuer le cours d'histoire romaine à l'université d'État de Gand en 1910, entraînant son retrait définitif de l'enseignement. Pendant et après cet épisode, il avait exprimé son mécontentement sur l'immixtion de la politique et de la religion dans la science. Comme l'a démontré Danny Praet récemment, il a même adopté, dans la première phase de sa vie, des positions anticléricales, quoique toujours d'une manière implicite $^{37}$. On ne s'attendrait donc pas à ce qu'il défende des savants confessionnels. Son caractère réservé et son souci d'une science libre où chacun pouvait exprimer son point de vue constituèrent certainement la base d'une telle attitude, ainsi que le respect qu'il avait pour les travaux de certains d'entre eux ${ }^{38}$. Reste que l'évolution de la position de Cumont n'est pas niable.

Du reste Cumont dénonce dans sa lettre le manque d'érudition de Couchoud qui, d'après lui, n'appartient pas au même univers scientifique que Loisy et lui-même: "Votre œuvre immense, d'une si haute tenue scientifique, mérite d'autres éloges que les louanges des mythologues ${ }^{39}$.» Il était pourtant conscient du fait que d'autres savants, comme Ernesto Buonaiuti et Loisy lui-même, ne refusèrent pas de collaborer avec Couchoud. On voit que Cumont éprouve un mépris profond pour le dilettantisme de Couchoud, qui n'avait pas une formation d'historien. De plus, Cumont se trouvait en désaccord total avec lui sur la thèse du Jésus mythique. On signalera que la bibliothèque privée de Cumont, conservée à Rome, ne contient pas d'exemplaire du Mystère de Jésus de Couchoud.

\section{BRUITS EN L'AIR?}

La réponse de Loisy aux accusations de Cumont ne tarda guère à venir. Étant donné que la lettre de Loisy à Cumont date du 6 juillet et

37. Danny Praet, «L'affaire Cumont: idéologies et politique académique à l'université de Gand au cours de la crise moderniste», in Corinne Bonnet, Danny Praet et alii (ed.), Science, politique et religion à l'époque de la crise moderniste, BHRI, Rome-Bruxelles, Brepols, à paraître (actes du colloque du 3 au 5 juin 2010).

38. Cumont a publié plusieurs comptes rendus positifs des travaux de savants religieux, comme par exemple du livre Les Martyrs de Dom H. Leclercq, Revue de l'Instruction publique en Belgique t. XLVI (1903), p. 400.

39. Cumont à Loisy, lettre du 3/07/1926, BnF, ms. NAF 15651, fo360. 
qu'on dispose d'une lettre de Couchoud répondant à Loisy, datée du 7 juillet, on peut conclure que les mots de Cumont avaient tellement ébranlé Loisy dans sa résolution que celui-ci jugea nécessaire d'écrire quasi simultanément à Couchoud pour le mettre à l'épreuve sur la base des renseignements obtenus par Cumont. Commençons par la lettre de Loisy à Cumont. Bien que Loisy donne raison à son ami, il ne le fait que partiellement. Apparemment, Loisy ne partage pas l'avis négatif de Cumont sur les promoteurs du Congrès. Il semble défendre Pulligny: «Je connais depuis près d'un quart de siècle le bailleur de fonds (...). Il m'a toujours voulu du bien.» Et il écrit à Cumont: «Couchoud est plus naïf qu'astucieux (...). Je ne pense pas du tout qu'il me prenne pour une vieille ganache dont on puisse faire l'homme sandwich d'une réclame, en flattant la vanitét ${ }^{40}$.»

Dans sa lettre, Loisy ne semble pas s'inquiéter de l'absence possible des savants religieux. Ensuite, il apparaît que, contrairement à Cumont, il sait parfaitement distinguer les intentions de Couchoud et de Pulligny de leurs convictions personnelles. Après sa défense de Couchoud et de Pulligny, Loisy prend l'offensive. En se retournant contre les informateurs de Cumont, il remarque avec amertume que les organisateurs attaqués avaient du moins fait l'effort de rendre hommage à son œuvre et à sa personne; il importe d'observer son irritation envers ceux qui avaient renseigné son ami sur le manque de neutralité de Couchoud, soulignant par là leur propre respectabilité et leur position prétendument indépendante :

Je n'ai pas à me louer autrement des savants respectables. Comme je ne demande rien à personne, je n'ai pas à me plaindre qu'on ne me donne rien. Mais il est des occasions qui parlent. Au dernier congrès d'histoire des religions ${ }^{41}$, par exemple, je me suis effacé volontairement, plus peut-être que je n'aurais dû; mais j'ai pensé voir qu'on m'effaçait encore un peu plus que je ne m'étais effacé moi-même ${ }^{42}$.

Comme la lettre de Couchoud date du 7 juillet, le «mythologue» doit avoir répondu immédiatement. Il y manifeste une fermeté

40. Loisy à Cumont, 6/07/1926, BnF, ms. NAF 15644, fo 282.

41 Il s'agit du congrès organisé par la Société Renan en 1923, où Loisy avait prononcé une communication sur le style rythmé du Nouveau Testament. Luimême raconte dans ses Mémoires qu'il n'y fit pas grande impression: A. Loisy (1931), p. 448.

42. Loisy à Cumont, 6/07/1926, BnF, ms. NAF 15644, fo 282. 
défensive qui trahit son agacement. Couchoud se défend contre les accusations transmises par Cumont et essaie de produire la preuve certaine de son désintéressement, donc de sa fiabilité, en s'abstenant de toute communication personnelle au Congrès ${ }^{43}$. Ensuite, il rectifie les renseignements concernant la position de la Société Renan. Paul Alphandéry, co-fondateur de la Société, aurait fait bon accueil au projet du Congrès. D'après Couchoud, Alphandéry aurait présenté le projet pendant les séances de la Société. Il semble que Couchoud dise la vérité puisque l'on retrouve effectivement l'annonce du Congrès Loisy dans la Revue de l'histoire des religions de l'époque ${ }^{44}$.

Les nouvelles indications de Couchoud, en contradiction flagrante avec celles de Cumont, semblent avoir plongé Loisy dans un abîme de perplexité. Cela se manifeste notamment dans sa correspondance où l'on retrouve les traces d'un deuxième courrier double à Cumont et à Couchoud ${ }^{45}$. Le 8 juillet Loisy envoie un petit mot à Cumont, montrant que les propos rassurants de Couchoud n'ont pas manqué de produire un certain effet:

Couchoud m'écrit qu'Alphandéry a fait distribuer le programme du Congrès à tous les membres de la Société Ernest Renan, et recommandé ledit Congrès à leur attention. La Société n'a pas à y prendre part officiellement. Je crois que Couchoud n'est pas si noir qu'on le fasse ${ }^{46}$.

Étonnamment, la lettre de Couchoud du 9 juillet ne porte aucune trace de l'apaisement manifesté par Loisy dans sa lettre à Cumont. Couchoud y reprend le ton défensif de la lettre antérieure, dont l'exacerbation implique que Loisy lui a probablement fait de nouveaux reproches, encore plus graves que les premiers. Sur un ton véritablement irrité, Couchoud passe à l'offensive tout en relevant un fait auquel Loisy s'était montré effectivement très sensible: "Quant aux gens qui me desservent auprès de vous, pourquoi n'ont-ils pas organisé eux-mêmes votre

43. Couchoud à Loisy, 7/07/1926, BnF, ms. NAF 15651, fo 37: «Puisque ma "mythologie" n'a pas votre agrément, j'étais bien décidé à la réserver pour une autre occasion".

44. RHR t. 93 (1926), p. 176; et le compte rendu de la séance de la Société Renan du 27 novembre 1926: RHR t. 95 (1927), p. 357.

45. Les lettres de Loisy à Couchoud n'étant probablement pas conservées, on déduit le courrier de Loisy à Couchoud de la lettre de Couchoud du 9 juillet, réponse à une lettre antérieure de Loisy.

46. Loisy à Cumont, 8/07/1926, cote 8082: Academia Belgica. 
Jubilé ? Pourquoi ne l'organiseraient-ils pas maintenant ${ }^{47}$ ?» En se plaignant dans sa lettre à Cumont du manque d'attention à l'égard de sa communication au Congrès de la Société Renan en 1923, Loisy avait déjà dénoncé l'attitude réservée des «savants respectables» de la Société envers son œuvre. Clairement, il se sentait mal aimé par les savants appartenant comme lui au secteur indépendant des sciences religieuses. Pleinement conscient de cette irritation, Couchoud prend son maître par son point faible en noircissant ses informateurs.

Dans sa lettre du 10 juillet 1926, qui est une réponse aux lettres des 7 et 8 juillet de Loisy, Cumont fait de nouvelles tentatives pour convaincre Loisy des mauvaises intentions de Couchoud. Tout d'abord, Cumont apparaît persuadé que Couchoud a menti à Loisy au sujet de la Société Ernest Renan:

Il se peut qu'A. ait fait distribuer le programme du congrès aux membres de la société Renan (bien que je ne l'aie pas reçu par lui) mais qu'il l'ait recommandé à leur adhésion, j'en doute beaucoup, car le même A., parlant à ma personne, m'a exprimé la crainte que le susdit congrès n'attirât surtout des gens peu qualifiés pour y prendre part et ne servît de prétexte à des manifestations déplacées ${ }^{48}$.

Paul Alphandéry aurait donc mené double jeu, se montrant d'une part sceptique à l'égard de Couchoud, en présence de Cumont, et d'autre part favorable au projet du Jubilé de Loisy, en présence de Couchoud ${ }^{49}$. Dans sa lettre Cumont ne répète pas ses préoccupations relatives aux savants catholiques et protestants, mais concentre son attention sur la position scientifique de Loisy. Avec sa douceur habituelle, il essaie surtout d'apaiser son ami agité :

Que les «savants respectables» ne vous aient pas suffisamment rendu justice ou du moins récompensé, je vous l'accorde... Que vous ayez un petit nombre d'amis, il se peut. Mais la raison n'en est-elle pas surtout que vous aimez la solitude? Et vous êtes plus grand dans votre splendide isolement au fond de votre tour d'ivoire, qu'en acceptant d'être fêté par d'infimes mythologues ${ }^{50}$.

47. Couchoud à Loisy, 9/07/1926, BnF, ms. NAF 15651, fo38.

48. Cumont à Loisy, 10/07/1926, BnF, ms. NAF 15651, fo 362.

49. Dans un des passages énigmatiques de ses Mémoires Loisy cite une phrase de cette lettre en remplaçant les «A.» d'Alphandéry par des «X»: «Le même $X$, parlant à ma personne, m'a exprimé la crainte que...»; Loisy (1931), p. 498.

50. Cumont à Loisy, 10/07/1926, BnF, ms. NAF 15651, f ${ }^{\circ} 362$. 
Même si Cumont blâme de nouveau le manque d'impartialité et d'érudition des «mythologues», envers son ami il se montre fin diplomate. Le «splendide isolement» et «la tour d'ivoire» sont en effet des expressions bien choisies pour indiquer la détermination avec laquelle Loisy a poursuivi son itinéraire scientifique. À la différence de Cumont, Loisy n'a pas construit sa tour d'ivoire par prédilection pour les sentiers peu fréquentés ${ }^{51}$, c'est-à-dire les sujets peu abordés dans l'histoire des religions, mais plutôt en se prononçant de manière très critique envers les grandes vérités généralement acceptées dans la discipline. En se dressant contre d'autres savants, soit catholiques (pensons au Père Lagrange), soit protestants (L'Évangile et l'Église dirigé contre Adolf von Harnack), soit encore d'autres convictions (les discussions continues avec Salomon Reinach), Loisy s'était effectivement taillé sa propre place scientifique. Puis Cumont précise de nouveau ces objections contre Couchoud, qui, à son avis, abuse de l'estime que beaucoup de savants ont pour la personne de Loisy, pour organiser un congrès dont lui-même serait le seul bénéficiaire ${ }^{52}$.

\section{LES PRISES DE POSITION DÉFINITIVES}

Malheureusement, nous ne disposons pas d'une réponse de Loisy qui nous permette d'évaluer sa réaction aux observations de Cumont. Cependant, il y a plusieurs autres sources qui laissent deviner ce qu'il advint. Dans ses Mémoires, Loisy se positionne clairement du côté de Couchoud et la correspondance entre les deux montre également sa décision finale ${ }^{53}$. La réponse de Couchoud à la lettre de Loisy, datée du 12 juillet, commence de la façon suivante: «C'est donc arrangé. Je continuerai à m'occuper de l'organisation matérielle de votre Jubilés ${ }^{54}$.» Loisy s'est résolu à s'engager dans le Congrès d'histoire du christianisme, malgré l'avis de Cumont. Peut-être la lettre de Cumont a-t-elle même accéléré cette décision.

51. Cumont à Loisy, 26/07/1911, BnF, ms. NAF 15651, fo 108 : «Je me suis toujours instinctivement détourné des sujets souvent traités; comme Callimaque, j'ai horreur de marcher sur les grandes routes et de boire aux fontaines publiques. »

52. Cumont à Loisy, 10/07/1926, BnF, ms. NAF 15651, f $\mathrm{f}^{\circ} 362$.

53. A. Loisy (1931), p. 498.

54. Couchoud à Loisy, 12/07/1926, BnF, ms. NAF 15651, f 39. 
Dans les lettres antérieures, on a remarqué la sensibilité que Loisy montrait envers les réactions négatives des «savants respectables» à l'organisation de son Jubilé et, sans le vouloir, Cumont a souligné leur duplicité en transmettant l'opinion très critique d'Alphandéry qui, d'après Couchoud, était au contraire favorable au Congrès (il y a en tout cas participé, avec une communication sur le christianisme du Moyen Âge).

Cette décision favorable à Couchoud n'implique pas pour autant que Loisy ait négligé entièrement les avertissements de son savant ami. La lettre de Couchoud démontre clairement que si Loisy a donné son consentement à la poursuite du Congrès, il ne l'a cependant pas fait inconditionnellement. Jusqu'à la fin du congrès, Loisy tint fermement les rênes. De plus, il prit la mesure des dangers qui auraient pu résulter d'une collaboration trop franche avec le camp de Couchoud. Apparemment, les restrictions de Loisy étaient relativement drastiques et elles portaient contre plusieurs catégories de savants. Dans sa lettre, Couchoud aborde ces exclusions voulues par Loisy par une moquerie caustique qui lui permet d'ironiser sur les agissements et intrigues des savants du camp indépendant:

Si je prenais au pied de la lettre quelques expressions de vos dernières lettres il y aurait à écarter les «scientistes», les «mythologues», les «anticléricaux» (les «amateurs», cela va de soi, doivent être soigneusement évités). C'est un programme difficile à remplir. Par exemple M. Guignebert, M. Mathiez, M. de Pulligny sont «anticléricaux»; M. van den Bergh v. Eysinga, M. Alfaric sont «mythologues»; Sir James Frazer, M. Kreglinger, M. Sartiaux sont «scientistes». Resteraient surtout les protestants et M. Brémond [sic]. Ce dernier, je pense, ne viendra pas et peut-être ne voudrez-vous pas donner à votre Jubilé une teinte trop protestante ${ }^{55}$.

Couchoud n'explicite pas les critères qui permettent de déterminer l'identité scientifique de chaque savant «indépendant», mais il fait écho à la prudence avec laquelle Loisy essaie d'éviter l'association de son nom aux autres savants non-catholiques. À juste titre, Couchoud attire l'attention sur la conséquence inévitable d'une telle attitude: en se distanciant démesurément du camp non-confessionnel, Loisy risque de s'entourer surtout de savants protestants, dont pourtant il s'est toujours soigneusement

55. Couchoud à Loisy, 12/07/1926, BnF, ms. NAF 15651, fo 39. Il n'y a, en effet, aucune trace de la présence de Bremond au Jubilé. 
tenu à distance; quant à l'abbé Henri Bremond, dont les travaux sur la mystique et la spiritualité modernes ne relèvent pas exactement de l'histoire du christianisme comme discipline, Couchoud doute de sa présence.

En même temps que, d'après les Mémoires, certains savants du côté confessionnel (catholiques ou protestants) commencent à faire de la contre-publicité en dissuadant leurs coreligionnaires de participer au Congrès ${ }^{56}$, Cumont se décide et parvient à la conclusion qu'il doit se retirer du Comité de patronage. Le 26 septembre, il écrit simultanément à Couchoud et à Loisy. Étant donné que Cumont a placé le brouillon de sa lettre à Couchoud entre les lettres reçues de ce dernier ${ }^{57}$, nous profitons de l'occasion unique d'évaluer les paroles de Cumont à ses deux correspondants. Même si la version définitive a certainement différé du brouillon, les mots souvent rayés et reformulés nous éclairent sur le contenu principal de la lettre de Cumont à Couchoud. Dans cette lettre, Cumont se présente de nouveau comme le défenseur des savants catholiques et protestants orthodoxes qu'il croit exclus. Il y précise de manière générale, mais très révélatrice, le rapport entre son objection à l'anticléricalisme de Couchoud et ses doutes sur sa scientificité :

L'histoire des religions [barré: est une science laïque] elle est née en dehors des églises [sic] et l'on comprend qu'on réunisse en un congrès purement laïc les savants qui s'en occupent. Mais les neuf dixièmes au moins des érudits qui [barré: applique leur érudition] se consacrent à l'histoire du christianisme [barré: de l'église (sic)] sont des catholiques ou des protestants orthodoxes: [barré: ils ne viendront], ni les uns ni les autres [barré: pour des mo] ne se rendront à la réunion [barré: à une réunion telle que celle] que vous projetez. Ou bien vous n'aurez qu'un petit nombre d'adhérents et votre congrès sera un simulacre de congrès, ou [barré: ce qui] pire encore, vous y admettrez des gens peu qualifiés comme savants et ce congrès prendra l'allure d'une simple manifestation anticléricale ${ }^{58}$.

D'après Cumont, l'histoire des religions et l'histoire du christianisme sont deux disciplines différentes avec leurs propres particularités. Tandis que l'histoire des religions est une science laïque, dans l'histoire du christianisme, la plus grande expertise

56. A. Loisy (1931), p. 523.

57. Après la lettre de Couchoud à Cumont, 27/09/1926, cote 8127 XL: Academia Belgica.

58. Cumont à Couchoud, cote $8127 \mathrm{XL}$ : Academia Belgica. 
se trouve au sein des institutions religieuses. Le dixième restant de ceux qui s'occupent de l'histoire du christianisme se divise en un petit groupe de savants compétents, d'une part, et en un groupe de personnes «peu qualifiées» et incompétentes, d'autre part. Personnellement, Cumont se consacre à l'histoire des religions, une science à distinguer de l'histoire du christianisme. Donc, il ne veut pas appartenir au Comité de patronage du Congrès d'histoire du christianisme. Du reste, il prévient Couchoud des dangers potentiels dérivant du fait que celui-ci refuse d'associer les savants religieux et choisit de collaborer avec le dixième des savants restants. Malgré les raisons évidentes que Cumont donne ici de son désengagement, la question reste ouverte de savoir pourquoi il n'a pas refusé son adhésion dès le début, si la distinction entre les deux disciplines était à ce point fondamentale à ses yeux. Il savait, par la première lettre de Couchoud, qu'Adolf von Harnack avait adhéré au Comité de patronage et que Couchoud, par conséquent, n'avait pas entièrement négligé les savants religieux de l'histoire du christianisme. En outre le discours sur l'incompétence de la majorité des savants non-religieux semble étrange, étant donné que Cumont avait du respect scientifique pour plusieurs d'entre eux qui ont étudié le christianisme. Les mots de Cumont portent presque à croire qu'il faut, à son avis, être chrétien pour être compétent dans l'étude du christianisme, ce qui serait d'ailleurs en contradiction avec l'activité de Cumont lui-même, qui a étudié, épisodiquement il est vrai, le christianisme sans être résolument chrétien $^{59}$.

Dans sa lettre à Loisy, Cumont ne détaille pas autant les raisons de son désengagement. D'une part, les dernières lettres de Cumont et Loisy ayant déjà montré leur divergence d'opinions, il est probable que Cumont n'a pas voulu pousser plus loin son désaccord avec Loisy. D'autre part, on peut aussi supposer qu'il était récalcitrant à l'idée de dévoiler l'identité de ses informateurs, que Loisy réduisit, selon toute probabilité, au groupe des «savants respectables». Dans sa lettre du 26 septembre Cumont explique donc à Loisy :

59. Sur le christianisme de Cumont, voir Corinne Bonnet, «Lux perpetua: un testament spirituel?», in Corinne Bonnet, Carlo Ossola, John Scheid (éd.), Rome et ses religions: culte, morale, spiritualité. En relisant Lux perpetua de Franz. Cumont, Mythos. Rivista di Storia delle Religioni suppl. 1 (2010), p. 136-138. 
Je viens encore d'apprendre sur le mythologue que vous savez des choses assez déplaisantes et je répugne à patronner une entreprise dont les dessous sont aussi suspects que ceux du congrès d'histoire du christianisme. Je viens donc d'écrire à ce mythologue que je le priais de ne plus faire figurer mon nom parmi les membres du comité60.

Le 27 septembre, Couchoud écrit à Cumont que son nom a été supprimé du Comité. Puisque Cumont n'a vitupéré qu'implicitement contre lui - contrairement aux mots adressés à Loisy au sujet du «mythologue»-, Couchoud ne semble pas se sentir visé par Cumont: "Les dangers que vous voulez bien signaler sont très réels. Nous arriverons, j'espère, à les éviter et à faire à $\mathrm{M}$. Loisy une fête jubilaire digne de lui ${ }^{61}$.» Bien que, dans sa lettre, Couchoud fasse valoir à juste titre le consentement final qu'a donné Loisy, la réponse de Loisy à Cumont démontre que Loisy restait réservé: dans sa lettre du 28 septembre 1926, il explique à Cumont qu'il en a assez des agissements autour du Congrès; il n'en tient pas entièrement son ami à l'écart:

J'ignore ce que vous avez pu en apprendre. Peu de choses bien certaines, probablement. Des adhésions on été reçues, mais très peu de promesses fermes pour les travaux. Dans ces conditions, il est très difficile de savoir quelle sera la physionomie du congrès en question. $\mathrm{Ne}$ connaissant pas les renseignements que vous avez ni leur source, il m'est très difficile de les apprécier. En ce qui me concerne, je me serais bien passé et je me passerais bien de ce congrès, qui, en mettant les choses au mieux, me rapportera beaucoup de fatigue, et en mettant les choses au pis, quelques ennuis ${ }^{62}$.

Le 3 octobre, Cumont annonce son arrivée à Paris et exprime le désir de rencontrer son ami. La lettre suivante datant du 7 mars 1927 renoue avec l'esprit cordial et les sujets habituels ${ }^{63}$. Il est assez probable qu'ils se sont effectivement rencontrés et ont pu aplanir leur désaccord de plus en plus profond sur les intentions de Couchoud, qui les avait éloignés lors de leurs intenses échanges épistolaires comme le démontre la dernière lettre de Loisy.

60. Cumont à Loisy, 26/09/1926, BnF, ms. NAF 15651, fo 364.

61. Couchoud à Cumont, 27/09/1926, cote 8127 XL: Academia Belgica.

62. Loisy à Cumont, 28/09/1926, BnF, ms. NAF 15644, fo 283.

63. Cumont à Loisy, 7/03/1927, BnF, ms. NAF 15651, f 368. 


\section{ÉPILOGUE ET CONCLUSION}

Le Congrès d'histoire du christianisme, à en croire Loisy et Marguerite Brunot (fille du doyen de la faculté des lettres de l'université de Paris), dès le début concernée par son organisation, reçut la visite de 350 personnes, avec des congressistes représentant 14 nationalités ${ }^{64}$.Pendant les trois jours du congrès, 48 communications furent présentées ${ }^{65}$. Sur les convictions religieuses des participants, on apprend par les Mémoires qu'il y avait aussi des savants catholiques, dont certains ne figurent pas sur la liste officielle des adhésions ${ }^{66}$. La participation active de Maurice Goguel, mais aussi de savants allemands comme Carl Clemen, prouve que les savants protestants ne renoncèrent pas à rendre hommage à Loisy.

Cumont décida en fin de compte de ne pas venir au Jubilé de son ami. Dans sa lettre du 7 mars, il prévient Loisy, en passant et sans donner d'explication: "J'ai oublié de vous demander quel jour du mois d'Avril [sic] on vous fêtait. Je voudrais m'associer de loin à cette manifestation puisque je ne puis être présent ${ }^{67}$. $\gg$ Cette décision ne surprend pas vu les distances qu'avait déjà prises Cumont en se retirant du Comité de patronage. Reste qu'on peut s'étonner qu'il n'ait pas trouvé le moyen d'assister comme auditeur au Congrès rendant hommage à son ami fidèle.

Le volume des actes, qui ne se trouve d'ailleurs pas dans la bibliothèque privée de Cumont, nous apprend que la thèse mythiste ne joua effectivement pas un rôle de premier plan au Congrès. Contrairement à la crainte de Cumont d'une manifestation orchestrée par Couchoud pour la promotion de ses propres thèses, l'organisateur et initiateur du Congrès maintint un profil bas. Loisy écrit même dans sa lettre du 22 mai 1927 à Cumont que Couchoud n'assista pas à une grande partie du Congrès car «il était allé pendant le carême faire des conférences mythologiques en Tunisie ${ }^{68} \gg$. Le Congrès ne fut pas la manifestation antichrétienne qu'avait craint Cumont. Même si Loisy fut flatté par l'initiative de Couchoud, il ne

64. A. Loisy (1931), p. 522-523, M. Brunot, article cité p. 40.

65. M. Brunot, article cité p. 42; A. Loisy (1931), p. 523 et p. 525-534.

66. A. Loisy (1931), p. 523: «Quelques adhérents catholiques avaient demandé que leurs noms ne fussent pas portés officiellement sur la liste.»

67. Cumont à Loisy, BnF, ms. NAF 15651, fo 368.

68. Loisy à Cumont, 22/05/1927, BnF, ms. NAF 15644, f 290. 
fut pas naïf et se donna beaucoup de peine pour garantir le caractère à son avis scientifiquement irréprochable du Congrès.

En étudiant la période préparatoire du Congrès d'histoire du christianisme, on a pu constater la grande complexité de l'entreprise. La correspondance entre Cumont, Loisy et Couchoud nous a permis d'être les témoins des désaccords profonds sur cette discipline et sur les personnes aptes à l'étudier. Distinguant catégoriquement les savants religieux des «théologiens laïcs» prêchant «leur foi négative», Cumont néglige en grande partie les compétences d'un groupe assez considérable de savants médiateurs, ni résolument chrétiens, ni antichrétiens. Cet oubli est difficile à comprendre dans la mesure où Loisy lui-même appartenait à cette mouvance. La pensée de Cumont, de même que les efforts effrénés de Loisy, Couchoud et les animateurs de la Société Ernest Renan afin de construire une image de neutralité illustrent la sensibilité extrême qui se manifestait lorsqu'il était question du christianisme et de son histoire. Dans les années 1920 l'histoire du christianisme était donc loin d'être une histoire parmi d'autres ${ }^{69}$.

annelies.lannoy@ugent.be

69. L'auteur exprime sa reconnaissance au FWO (Fonds voor Wetenschappelijk Onderzoek - Vlaanderen/Fonds de la Recherche Scientifique - Flandre) qui a financé le projet de recherche G.0126.08 sur l'origine du christianisme dans la pensée de F. Cumont et A. Loisy, dont le présent travail est issu; à l'université de Gand, à Danny Praet pour ses observations nombreuses qui ont profondément enrichi ce texte et à Corinne Bonnet pour sa relecture précieuse. 